ICRR-Report-345-95-11

UT-730

\title{
Can Decaying Particles Raise the Upperbound on the Peccei-Quinn Scale?
}

\author{
M. Kawasaki \\ Institute for Cosmic Ray Research, University of Tokyo, Tanashi 188, Japan \\ T. Moroi \\ Theoretical Physics Group, Lawrence Berkeley Laboratory, University of California, \\ Berkeley, CA 94720, U.S.A. \\ T. Yanagida \\ Department of Physics, University of Tokyo, Tokyo 113, Japan
}

\begin{abstract}
We have reexamine the effect of entropy production on the cosmic axion density and find that the Peccei-Quinn scale $F_{a}$ larger than about $10^{15} \mathrm{GeV}$ is not allowed even if large entropy is produced by the decays of coherent oscillations or nonrelativistic massive particles. We stress that this result is independent of the details of models for the decaying particles.
\end{abstract}




\section{Introduction}

The axion [1, 2, 3, 国 is the Nambu-Goldstone boson associated with the Peccei-Quinn symmetry breaking which was invented as a natural solution to the strong CP problem in QCD [5]. The breaking scale $F_{a}$ of Peccei-Quinn symmetry is stringently constrained by laboratory experiments, astrophysics and cosmology. The consideration of cooling processes due to the axion emission in red giants and SN1987A requires that $F_{a}$ should be greater than about $10^{10} \mathrm{GeV}[6]$. On the other hand, $F_{a}$ should be less than about $10^{12} \mathrm{GeV}$ so that the energy density of the coherent oscillations of the axion field should be less than the critical density of the universe [7]. Thus the allowed range of $F_{a}$ is given between $10^{10} \mathrm{GeV}$ and $10^{12} \mathrm{GeV}$ which is called the axion window.

However, Steinhardt and Turner [8] showed that the entropy production due to the first order phase transition or the out-of-equilibrium decays of massive particles dilutes the axion density and makes the upperbound on $F_{a}$ as large as $10^{18} \mathrm{GeV}$. The upperbound $\left(F_{a} \lesssim 10^{18} \mathrm{GeV}\right)$ was obtained by requiring the entropy production should not dilute the baryon density too much, i.e. the dilution factor should be less than $10^{6}$ assuming the initial baryon-to-entropy ratio to be at most $10^{-4}$.

However, in the case of the decaying particles, the analysis in ref. [8] is unsatisfactory since the authors assumed the radiation dominated universe when the axion field starts to oscillate. As we will show below, it is more reasonable to consider that the universe is already decaying-particle dominated at that epoch. Therefore, we reexamine the entropy production from the decays of coherent oscillations or light particles and obtain the upperbound, $F_{a} \lesssim 10^{15} \mathrm{GeV}$, by imposing the reheating temperature to be less than $1 \mathrm{MeV}$ in order to keep the success for the primordial nucleosynthesis in the big-bang cosmology. We stress that this result is independent of detailed models for the decaying particles. 


\section{Cosmological Evolution}

Let us consider the cosmological evolution of the axion field and the coherent oscillation of the field $\phi$ with potential $m_{\phi}^{2} \phi^{2} / 2$ and density $\rho_{\phi}$. This coherent oscillation is equivalent to the non-relativistic decaying particle with the same energy density. Therefore, we only consider the coherent oscillation hereafter. We assume that $\rho_{\phi}$ dominates the energy density of the universe when the oscillation of the axion starts. If the universe is radiationdominated, the axion starts to oscillate at $T \simeq 1 \mathrm{GeV}$. In this case the entropy production factor is given by $\sim\left[\left(T_{R}^{4} / T_{1}^{4}\right)\left(a\left(T_{R}\right) / a\left(T_{1}\right)\right)^{4}\right]^{3 / 4} \simeq\left(T_{R} / T_{1}\right)^{3}\left(\rho_{\phi}\left(T_{1}\right) / T_{R}^{4}\right) \lesssim\left(T_{1} / T_{R}\right)$, where $a$ is the scale factor of the universe, $T_{R}$ the temperature just after the $\phi$ decay and $T_{1}$ the temperature at which the axion field starts to oscillate. Since $T_{R}$ should be greater than $1 \mathrm{MeV}$ to keep the success of primordial nucleosynthesis, the entropy production factor becomes less than $O\left(10^{3}\right)$. Therefore, the $\phi$-dominated universe is a good assumption as far as the large entropy production (with the entropy production factor greater than $\left.O\left(10^{3}\right)\right)$ is considered.

The axion starts to oscillate at $t=t_{1}$ when $3 H \simeq m_{a}$. Thus at $t=t_{1}$,

$$
\rho_{\phi}\left(t_{1}\right) \simeq \frac{m_{a}\left(T_{1}\right)^{2} M^{2}}{3}
$$

where $M=2.4 \times 10^{18} \mathrm{GeV}$ is the gravitational mass and $m_{a}(T)$ is the axion mass which depends on the temperature $T$ as 9$]$

$$
m_{a}(T) \simeq \begin{cases}0.1 m_{a}\left(\Lambda_{\mathrm{QCD}} / T\right)^{3.7} & \text { for } T \gtrsim \Lambda_{\mathrm{QCD}} / \pi \\ m_{a} & \text { for } T \lesssim \Lambda_{\mathrm{QCD}} / \pi\end{cases}
$$

where $\Lambda_{\mathrm{QCD}} \simeq 0.2 \mathrm{GeV}$, and $m_{a}$ is the axion mass at $T=0$.

Until the coherent $\phi$ oscillation decays, the ratio of the axion- to $\phi$-number densities stays constant. Therefore, the ratio of the energy density of the axion $\rho_{a}$ to that of $\phi, \rho_{\phi}$, is expressed as

$$
\frac{\rho_{a}}{\rho_{\phi}}=\frac{m_{a}}{m_{\phi}} \frac{m_{a}\left(T_{1}\right) F_{a}^{2} \theta^{2}}{\rho_{\phi}\left(t_{1}\right) / m_{\phi}}=\frac{3}{2} \frac{F_{a}^{2} \theta^{2}}{M^{2}} \frac{1}{\xi\left(T_{1}\right)},
$$

where $\theta \sim O(1)$ is the initial axion amplitude in units of $F_{a}$ and $\xi\left(T_{1}\right) \equiv m_{a}\left(T_{1}\right) / m_{a} \leq 1$.

${ }^{*} \theta$ takes $\pi / \sqrt{3}$ in the non-inflationary universe [9]. 
The $\phi$ decay occurs when $3 H \simeq \Gamma_{\phi}$ where $\Gamma_{\phi}$ is the decay rate of $\phi$. The cosmic temperature $T_{R}$ just after the decay is given by

$$
T_{R} \simeq\left(\frac{10}{\pi^{2} g_{*}}\right)^{1 / 4} \sqrt{M \Gamma_{\phi}}=0.55 \sqrt{M \Gamma_{\phi}},
$$

where $g_{*}$ is the effective number of massless degrees of freedom and we have taken $g_{*}=$ 10.75. The entropy density just after the decay is given by

$$
s\left(T_{R}\right)=\frac{2 \pi^{2}}{45} g_{*} T_{R}^{3} .
$$

Since the energy density of the coherent $\phi$ oscillation just before the decay is $M^{2} \Gamma_{\phi}^{2} / 3$, the axion density at the decay epoch becomes

$$
\rho_{a}\left(T_{R}\right) \simeq\left(\frac{\rho_{a}}{\rho_{\phi}}\right) \rho_{\phi}\left(T_{R}\right) \simeq 5.3 T_{R}^{4} M^{-2} F_{a}^{2} \theta^{2} \xi\left(T_{1}\right)^{-1} .
$$

Then we can estimate the axion-to-entropy ratio as

$$
\frac{\rho_{a}}{s} \simeq 1.1 T_{R} F_{a}^{2} \theta^{2} M^{-2} \xi\left(T_{1}\right)^{-1} .
$$

This value should be compared with the ratio of the present values of the critical density $\rho_{c r, 0}$ to the entropy density $s_{0}$, which is given by

$$
\frac{\rho_{c r, 0}}{s_{0}} \simeq 3.6 \times 10^{-9} h^{2} \mathrm{GeV},
$$

where $h$ is the present Hubble constant in units of $100 \mathrm{~km} / \mathrm{sec} / \mathrm{Mpc}$. Then, the density parameter of axion $\Omega_{a}$ is expressed as

$$
\begin{aligned}
\Omega_{a} h^{2} & \simeq 3.1 \times 10^{8} \mathrm{GeV}^{-1} T_{R} F_{a}^{2} \theta^{2} M^{-2} \xi\left(T_{1}\right)^{-1} \\
& \simeq 5.3\left(\frac{T_{R}}{1 \mathrm{MeV}}\right)\left(\frac{F_{a} \theta}{10^{16} \mathrm{GeV}}\right)^{2} \xi\left(T_{1}\right)^{-1}
\end{aligned}
$$

$\dagger$ This formula (7) is applicable for $T_{R} \lesssim 1 \mathrm{GeV}$. 


\section{Constraint on $F_{a}$}

Since the entropy production should occur before the primordial nucleosynthesis, $T_{R}$ should be higher than about $1 \mathrm{MeV}$. Then, from eq.(四), we get $\Gamma_{\phi} \gtrsim 1.3 \times 10^{-24} \mathrm{GeV}$. Requiring $\Omega_{a} h^{2} \lesssim 1$ and taking $\xi \leq 1$ into account, we can obtain the upper limit on $F_{a}$ from eq.(9):

$$
F_{a} \lesssim 4.4 \times 10^{15} \theta^{-1} \mathrm{GeV} .
$$

The above constraint might be more stringent if the cosmic temperature at $t_{1}$ is greater than about $0.1 \mathrm{GeV}$ and hence $\xi \ll 1$. Therefore, we need to estimate $T_{1}$ and $\xi\left(T_{1}\right)$. For this end, we must take account of the fact that the temperature decreases as $T \propto a^{-3 / 8}$. Using $a\left(T_{R}\right) / a\left(T_{1}\right) \simeq\left(\rho_{\phi}\left(T_{R}\right) / \rho_{\phi}\right)^{-1 / 3} \simeq\left(m_{a}\left(T_{1}\right) / \Gamma_{\phi}\right)^{2 / 3}$,

$$
\frac{T_{1}}{T_{R}} \simeq\left(\frac{m_{a}\left(T_{1}\right)}{\Gamma_{\phi}}\right)^{1 / 4}
$$

From eqs.(国), (田) and (11), $T_{1}$ is given by

$$
T_{1} \simeq 0.07 \mathrm{GeV}\left(\frac{T_{R}}{1 \mathrm{MeV}}\right)^{0.26}\left(\frac{F_{a}}{10^{15} \mathrm{GeV}}\right)^{-0.13} .
$$

When $T_{R} \simeq 1 \mathrm{MeV}$ and $F_{a} \simeq 10^{15} \mathrm{GeV}, T_{1}$ is about $0.1 \mathrm{GeV}$. At such low temperatures, the axion mass is almost equal to its zero-temperature value, i.e. $m_{a}$. Therefore, $\xi\left(T_{1}\right) \simeq 1$ and the constraint $(10)$ is correct.

\section{Conclusion}

We have reexamined the effect of the large entropy production on the axion density in the early universe and have found that the Peccei-Quinn scale $F_{a}$ larger than about $10^{15} \mathrm{GeV}$ is not allowed. This upperbound is three orders of magnitude larger than that without entropy production but much smaller than the previous estimation [8]. In the present analysis, we have assumed that the universe is dominated by the coherent oscillation or

${ }^{\ddagger}$ Note that the decay does not occur instantaneously. During the $\phi$ decay, the temperature does not decrease as $a^{-1}$ due to the heating effect of the decay. For details, see ref. [9]. 
the decaying particle when the axion starts to oscillate. On the other hand the radiationdominated universe was assumed in the previous work. However, when the entropy is increased by a factor greater than $O\left(10^{3}\right)$, our assumption is correct. On the other hand, in the case where the entropy production factor is less than $O\left(10^{3}\right)$, the upperbound on Peccei-Quinn scale $F_{a}$ is raised up by $O\left(10^{3}\right)$ at most which completes our conclusion.

We have not discussed the dilution of the cosmological baryon number asymmetry because it depends on the details of the models for baryogenesis. For example, if we adopt the Affleck-Dine mechanism for baryogenesis [10], the $\phi$ decay with the reheating temperature about $1 \mathrm{MeV}$ is consistent with the observed baryon number of the universe [11]. 


\section{References}

[1] R.D. Peccei and H.R. Quinn, Phys. Rev. Lett. 38 (1977) 1440.

[2] F. Wilczeck, Phys. Rev. Lett. 40 (1978) 279.

[3] J.E. Kim, Phys. Rev. Lett. 43 (1979) 103;

M. Shifman, A. Vainshtein and V. Zakharov, Nucl. Phys. B166 (1980) 493.

[4] M. Dine, W. Fischler and M. Srednicki, Phys. Lett. B104 (1981) 199.

[5] G. 't Hooft, Phys. Rev. Lett. 37 (1976) 8.

[6] G. Raffelt, Phys. Rep. 198 (1990) 1;

M.S. Turner, Phys. Rep. 197 (1990) 67.

[7] J. Preskill, M. Wise and F. Wilczek, Phys. Lett.B180 (1983) 127;

L. Abbott and P. Sikivie, Phys. Lett.B180 (1983) 133;

M. Dine and W. Fischer, Phys. Lett.B180 (1983) 137.

[8] P.J. Steinhardt and M.S. Turner, Phys. Lett. B129 (1983) 51.

[9] E.W. Kolb and M.S. Turner, The Early Universe, Addison-Wesley (1990).

[10] I. Affleck and M. Dine, Nucl. Phys. B249 (1985) 361.

[11] T. Moroi, M. Yamaguchi and T. Yanagida, Phys. Lett. B342 (1995) 105. 